\title{
Severe Cross-Modal Object Recognition Deficits in Rats Treated Sub-Chronically with NMDA Receptor Antagonists are Reversed by Systemic Nicotine: Implications for Abnormal Multisensory Integration in Schizophrenia
}

\author{
Derek L Jacklin', Amit Goel', Kyle J Clementino', Alexander WM Hall', John C Talpos² and \\ Boyer D Winters*,I \\ 'Department of Psychology and Collaborative Neuroscience Program, University of Guelph, Guelph, ON, Canada; ${ }^{2}$ Translational Research, \\ Janssen Pharmaceutical Companies of Johnson \& Johnson, Beerse, Belgium
}

\begin{abstract}
Schizophrenia is a complex and debilitating disorder, characterized by positive, negative, and cognitive symptoms. Among the cognitive deficits observed in patients with schizophrenia, recent work has indicated abnormalities in multisensory integration, a process that is important for the formation of comprehensive environmental percepts and for the appropriate guidance of behavior. Very little is known about the neural bases of such multisensory integration deficits, partly because of the lack of viable behavioral tasks to assess this process in animal models. In this study, we used our recently developed rodent cross-modal object recognition (CMOR) task to investigate multisensory integration functions in rats treated sub-chronically with one of two N-methyl-D-aspartate receptor (NMDAR) antagonists, MK-80I, or ketamine; such treatment is known to produce schizophrenia-like symptoms. Rats treated with the NMDAR antagonists were impaired on the standard spontaneous object recognition (SOR) task, unimodal (tactile or visual only) versions of SOR, and the CMOR task with intermediate to long retention delays between acquisition and testing phases, but they displayed a selective CMOR task deficit when mnemonic demand was minimized. This selective impairment in multisensory information processing was dose-dependently reversed by acute systemic administration of nicotine. These findings suggest that persistent NMDAR hypofunction may contribute to the multisensory integration deficits observed in patients with schizophrenia and highlight the valuable potential of the CMOR task to facilitate further systematic investigation of the neural bases of, and potential treatments for, this hitherto overlooked aspect of cognitive dysfunction in schizophrenia.

Neuropsychopharmacology (2012) 37, 2322-233I; doi: I0.I038/npp.20 I2.84; published online 6 June 2012
\end{abstract}

Keywords: cross modal; ketamine; MK-80I; spontaneous object recognition; tactile; visual

\section{INTRODUCTION}

Cognitive dysfunction, which is often apparent before the onset of psychosis, is a core component of schizophrenia. Indeed, the MATRICS (Measurement and Treatment Research to Improve Cognition in Schizophrenia) initiative has recently outlined eight cognitive domains commonly disrupted in schizophrenia: processing speed, attention/vigilance, working memory, verbal learning and memory, visual learning and memory, reasoning and problem solving, verbal comprehension, and social cognition (Nuechterlein et al, 2004). Thus, the cognitive deficits in schizophrenia are varied and complex, and there is much interest in elucidating the neural bases of these impairments.

*Correspondence: Dr BD Winters, Department of Psychology, University of Guelph, Guelph, ON, Canada NIG 2WI, Tel: +519 8244120 (52163), Fax: + 519837 8629, E-mail: bwinters@uoguelph.ca Received 15 January 2012; revised 30 April 2012; accepted 30 April 2012
Several animal models have been developed to study the bases of the neuro- and psychopathology of schizophrenia. One of the most common classes of these is based on the $\mathrm{N}$-methyl-D-aspartate receptor (NMDAR) hypofunction hypothesis (Jentsch and Roth, 1999; Neill et al, 2010), which emerged from findings that acute administration of non-competitive NMDAR antagonists such as phencyclidine and ketamine induces schizophrenia-like symptoms in healthy humans and exacerbates symptoms in patients with schizophrenia (Itil et al, 1967; Luby et al, 1959). Accordingly, rodents and nonhuman primates treated with non-competitive NMDAR antagonists display behavioral features consistent with many symptoms of schizophrenia (Gilmour et al, 2011). Repeated dosing with these drugs may provide greater validity than acute administration, as such regimens induce neurochemical changes similar to those seen in schizophrenic patients (Cochran et al, 2003; Enomoto et al, 2005; Reynolds et al, 2004), as well as lasting cognitive deficits that can be observed when animals are tested in a drug-free state (Jentsch and Roth, 1999). 
Indeed, sub-chronic treatment with PCP or ketamine has been reported to decrease GABAergic markers and glucose utilization in the rat prefrontal cortex (Cochran et al, 2003; Pratt et al, 2008), consistent with observed prefrontal dysfunction in patients with schizophrenia (Lewis and Gonzalez-Burgos, 2008).

In accord with the visual learning and memory domain identified by MATRICS, object recognition is commonly impaired in rats treated with non-competitive NMDAR antagonists (Lyon et al, 2011). Standard rodent object recognition tasks, however, are not purely visual in nature, and object representation typically involves integration of information across multiple sensory modalities. Although sensory processing deficits in schizophrenia have long been acknowledged (Adler et al, 1982; Braff, 1989; Ford et al, 2004), the integration of information from different sensory modalities has only recently begun to attract greater attention (Stone et al, 2011; Williams et al, 2010). The ability to form comprehensive multimodal object representations is crucial to developing accurate percepts and memories about the environment, and much of our everyday information processing is facilitated by the ability to combine information from more than one sensory source. Indeed, research indicates that the presentation of congruent information from different sensory modalities can enhance information processing in healthy human participants (Stein and Meredith, 1993). A recent study, however, showed that such a facilitative effect was significantly attenuated in patients with schizophrenia (Williams et al, 2010). Furthermore, incongruent information from different modalities can cause perceptual illusions, such as the audio-visual McGurk effect (McGurk and MacDonald, 1976), but there is evidence that schizophrenic patients may be less susceptible to such illusions (Pearl et al, 2009). Additional research suggests that patients with schizophrenia display decreased multisensory integration for complex stimuli, such as emotional faces and voices (de Gelder et al, 2005; de Jong et al, 2009) and speech-related stimuli (de Gelder et al, 2003; Ross et al, 2007). Thus, there is a growing need for a better understanding of the nature of these apparent multisensory deficits in schizophrenia.

The relative lack of data regarding the neural bases of this cognitive impairment is partly related to the limited availability of viable tests to study multisensory integration in animal models. Indeed, the importance of multisensory integration was recognized by the first CNTRICS (Cognitive Neuroscience Treatment Research to Improve Cognition in Schizophrenia) breakout session, which suggested the inclusion of 'cross-modal integration' in a preclinical battery for cognition in schizophrenia research (Butler et al, 2008). We have recently introduced a cross-modal (tactile-to-visual) variant of the rodent spontaneous object recognition (SOR) task that enables assessment of multisensory integration (Winters and Reid, 2010). The aim of this study was, therefore, to evaluate the performance of rats treated with sub-chronic regimens of either MK-801 or ketamine in the standard SOR task, the cross-modal object recognition (CMOR) task, and two unimodal variants of SOR (tactile- and visual-only). We predicted that the CMOR task would be particularly sensitive to cognitive deficits in these animals. Finally, to further validate the CMOR task, we assessed the ameliorative potential of systemic nicotine. Nicotine can improve schizophrenia-associated cognitive impairment, and changes in nicotinic acetylcholine receptors are associated with the neuropathophysiology of schizophrenia (Leonard et al, 2000; Woodruff-Pak and Gould, 2002).

\section{MATERIALS AND METHODS}

\section{Subjects}

The subjects were 119 adult male Long Evans rats (Charles River, Quebec), weighing $\sim 300-350 \mathrm{~g}$ at the onset of experimentation. Rats were housed in pairs and maintained on a reverse light/dark cycle (0700 hours lights off; 1900 hours lights on). All behavioral testing occurred during the dark phase of the cycle, and lighting conditions in the testing room were specific to the task being run (see below). Rats received $20 \mathrm{~g}$ of rodent chow each evening to maintain $85-90 \%$ of free-feeding body weight during behavioral testing. Water was available ad libitum throughout the experiments. All procedures adhered to the guidelines of the Canadian Council on Animal Care and were approved by the Animal Care Committee at the University of Guelph.

\section{Sub-chronic Drug Administration}

All rats were handled for 1 week before the commencement of drug administration and then randomly assigned to treatment groups. Sub-chronic MK-801 ( + )-5-methyl-10, 11-dihydro-SH-dibenzo(a,d)cyclohepten-5,10-imine maleate; dizocilpine) or ketamine were administered according to established protocols (Beninger et al, 2009; Enomoto and Floresco, 2009). Rats in the drug conditions received twice daily i.p. injections of MK-801 (0.5 mg/kg in saline; Sigma) or ketamine $(30 \mathrm{mg} / \mathrm{kg}$; Bioniche, Belleville, ON, Canada) at a volume of $1 \mathrm{ml} / \mathrm{kg}$ of body weight at approximately 0800 hours and 2000 hours for seven (MK-801) or 10 (ketamine) consecutive days. Control rats received i.p. injections of physiological saline $(0.9 \% \mathrm{NaCl}, \mathrm{pH} 7.0$; Sigma $)$ at the same times. Experimenters were blind to the identity of the drug being administered. The drug administration phase was followed by a 7- or 10-day-washout period for MK-801 and ketamine, respectively. Animals remained undisturbed in their home cages on free feed during the washout phase.

\section{Object Recognition Testing}

At the end of the washout period, object recognition testing began. All object recognition tasks were conducted in a Y-shaped apparatus as described previously (Forwood et al, 2005; Winters et al, 2004; Winters and Reid, 2010). Duplicate copies of objects made from plastic, ceramic, glass, and aluminum and ranging in height from 10 to $20 \mathrm{~cm}$ were used. The different objects varied with respect to their visual and tactile qualities. All objects were affixed to the floor of the apparatus with a reusable adhesive putty to prevent them from being displaced during testing. Before being placed in the apparatus, objects were wiped with $50 \%$ ethanol. 
Each experiment followed the same general procedure. For all experiments, the experimenter was blind to the group assignments of subjects. All rats were habituated to the Y-shaped apparatus over 2 consecutive days. For the standard SOR experiments, these habituation sessions consisted of two 5-min exposures to the empty Y-shaped apparatus in normal (white) lighting conditions. For the CMOR and unimodal SOR tasks, each rat spent $5 \mathrm{~min}$ in the empty Y-shaped apparatus in red light and $5 \mathrm{~min}$ in the apparatus in white light with transparent plastic barriers in place; the order of these exposures was counterbalanced across animals.

Behavioral testing began $24 \mathrm{~h}$ after the second habituation session. Each trial consisted of two phases, sample (acquisition) and choice (test), separated by a retention delay. To reduce variability, rats were run on two trials in each condition for each experiment, with at least $24 \mathrm{~h}$ separating the end of one trial from the beginning of the next and with all conditions counterbalanced across the total number of trials (see specific details below). Habituation to the Y-shaped apparatus was not repeated before each trial. A different object pair was used for each trial for a given rat, and the order of exposure to object pairs as well as the designated sample and novel objects for each pair were counterbalanced.

In the sample phase, two identical objects were placed in the Y-shaped apparatus, one at the end of each exploration arm. The time spent exploring the two objects was scored by an experimenter viewing the rat on a video screen. Exploration of an object was defined as directing the nose to the object, or the area of the transparent barrier within the outline of the object in the visual-only conditions, at a distance of $<2 \mathrm{~cm}$ and/or touching it with the nose or whiskers. The sample phase ended when the rat had explored the identical objects for a total of $25 \mathrm{~s}$, or after 3 min had passed, whichever came first.

In the choice phase, the Y-shaped apparatus contained the sample object in one arm and a new object in the other. The exploration arms in which the choice objects were placed were counterbalanced between rats and across trials. The time spent exploring the novel and familiar objects was recorded for the 1-min choice phase. We then calculated a discrimination ratio, the difference in time spent exploring the novel and familiar objects divided by the total time spent exploring the objects, for each object recognition trial. This measure takes into account individual differences in the total amount of exploration time. Normal rats tend to explore the novel object more than the familiar sample object in this SOR paradigm.

Figure 1 illustrates the various versions of the SOR task used in this study. For the standard SOR task, rats were given full visual and tactile access to the objects in both the sample and choice phases. For tactile (unimodal) SOR, the sample and choice phases were run in red light to limit exploration to the tactile features of the objects, as rat vision is severely compromised in red light (Jacobs et al, 2001; Winters and Reid, 2010). For visual (unimodal) SOR, the sample and choice phases were run in white light with a transparent plastic barrier between the rats and the objects to limit exploration to the visual features of the objects. For the CMOR task, the sample phase was run in red light, and the choice phase was run in white light with the transparent

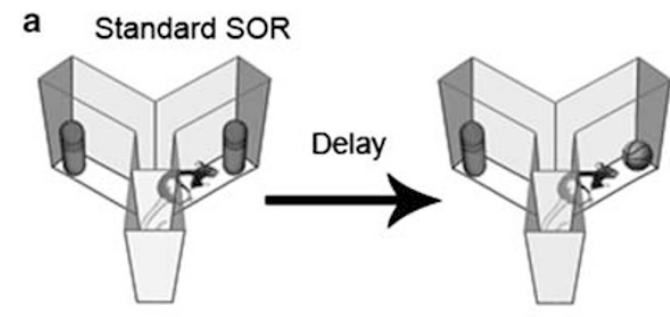

b Tactile SOR

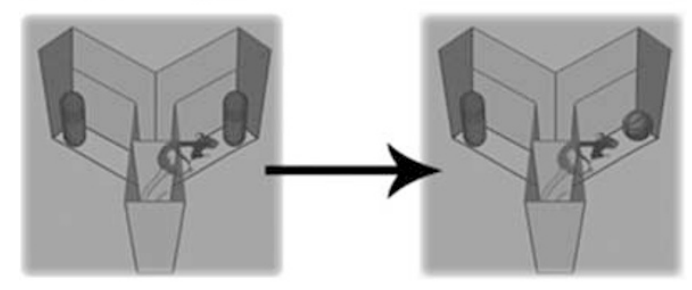

c Visual SOR

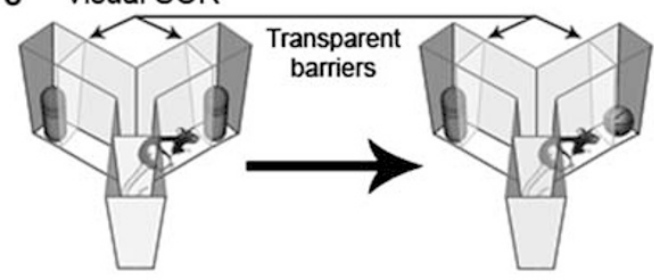

d $\mathrm{CMOR}$

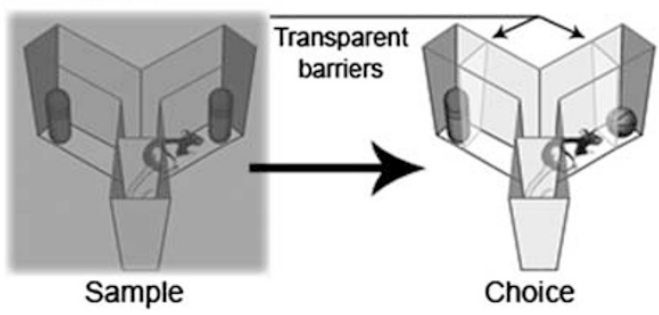

Figure I Illustration of the four spontaneous object recognition (SOR) tasks used in this study. (a) In the standard SOR task, both the sample and choice phases are run in white light, allowing full access to the tactile and visual properties of objects. The retention delays for SOR in this study were 'immediate', $5 \mathrm{~min}$, or $24 \mathrm{~h}$. For all other tasks, the delays were 'immediate' or I h. (b) In the tactile SOR task, the sample and choice phases are run in red light, permitting only the tactile qualities of the objects to be explored. (c) In the visual SOR task, the sample and choice phases are run in white light with transparent barriers in front of the objects. These barriers prevent physical contact, allowing rats to explore only the visual characteristics of the objects. (d) In the cross-modal object recognition (CMOR) task, the tactile sample phase is run in red light, whereas the visual choice phase is run in white light with transparent barriers in front of the objects. This requires rats to use the visual information available during the choice phase to recognize an object based on a tactile representation previously acquired in the sample phase.

barriers, thereby necessitating a tactile-to-visual CMOR judgement on the part of the rats (see Winters and Reid, 2010 for further details).

Four types of experiments were run with each NMDA receptor antagonist preparation:

(1) Standard SOR with multiple delays ('immediate', $5 \mathrm{~min}$, and $24 \mathrm{~h}$ ). The immediate retention delay was the length of time require for the experimenter to remove the sample objects and replace them with the choice 
pairing; this took $\sim 45 \mathrm{~s}$ on average. Two separate batches of 20 rats ( $10 \mathrm{MK}-801 / 10$ saline; 10 ketamine/10 saline) were run in standard SOR testing with the three retention delays counterbalanced across trials. Thus, each rat was run for a total of six trials (two trials per delay length), with a minimum of $24 \mathrm{~h}$ separating trials.

(2) 1-h delay CMOR, tactile SOR, and visual SOR. Two new batches of rats (9 MK-801/10 saline; 20 ketamine/20 saline) were run with counterbalanced trials of the CMOR, tactile SOR, and visual SOR tasks, all with a 1-h retention delay. Thus, each rat was run for a total of six trials (two trials per task type), with a minimum of $24 \mathrm{~h}$ between trials.

(3) Immediate delay CMOR, tactile SOR, and visual SOR. Two batches of rats (9 MK-801/10 saline; 10 ketamine/ 10 saline) were run with counterbalanced trials of the CMOR, tactile SOR, and visual SOR tasks, all with the immediate retention delay. The $19 \mathrm{MK}-801 /$ saline rats were the same rats tested with the 1-h delay, above. Each rat was run for a total of six trials (two trials per task type), with a minimum of $24 \mathrm{~h}$ separating trials.

(4) Immediate delay CMOR with nicotine. Two batches of rats (9 MK-801/10 saline; 10 ketamine/9 saline) were run in probe trials with acute i.p. nicotine $((-)$-nicotine hydrogen tartrate salt, $0.2 \mathrm{mg} / \mathrm{kg}$; Sigma) or saline injections administered $20 \mathrm{~min}$ before the sample phase in the CMOR task with the immediate retention delay. Order of acute drug administration was counterbalanced, and experimenters were blind to the identity of the drugs being administered. The MK-801/saline rats were the same rats tested with the $1 \mathrm{~h}$ and immediate delays, above. The ketamine/saline rats were the same as those tested with the immediate delay, above (one rat from the saline group died between experiments). All rats were run for four total trials (two with saline and two with nicotine) with a minimum of $48 \mathrm{~h}$ separating trials.

Following the nicotine probe trials, a new batch of rats was prepared to assess the dose-response function of systemic nicotine in naïve animals. A total of 10 new rats were treated with sub-chronic MK-801 as described above, and 10 additional rats received sub-chronic saline administration. Following the 7-day-washout period, these new groups were assessed in the immediate delay version of the CMOR task with counterbalanced administration of physiological saline and three doses of nicotine $(0.05,0.2$, and $0.8 \mathrm{mg} / \mathrm{kg}$ ) to each rat. Thus, these rats were naïve at the start of nicotine testing. Each rat was run for a total of four trials, one with each dose of nicotine and saline. Experimenters were blind to the identity of the drug being administered, and order of drug administration was counterbalanced within groups across these four trials. A minimum of 48 -h separated trials, and object sets were not repeated for a given rat within the four trials of CMOR testing.

\section{Data Analysis}

Group means of three measures taken from object recognition testing were analyzed for each experiment: the total object exploration in the sample phase, the total object exploration in the choice phase, and the discrimination ratio, which is the index of object recognition ability in these tasks. The total exploration control measures were analyzed to assess the possibility of gross differences in general exploratory behavior. Group means for these three measures were submitted to two-way mixed factorial ANOVAs with group as a between-subjects factor and delay (standard SOR experiments: immediate, $5 \mathrm{~min}$, or $24 \mathrm{~h}$ ), task (CMOR, tactile, or visual), or drug (nicotine dose or saline) as a within-subjects factor in the respective experiments; post-hoc independent samples $t$-tests were used to further assess group differences. An additional analysis was performed on the object recognition data for each experiment using independent samples $t$-tests to compare the mean discrimination ratio in each condition to a score of 0 (ie, no significant discrimination between the novel and familiar objects in the choice phase). All statistical analyses were conducted with a significance level of $\alpha=0.05$ using SPSS 19.0 for Windows.

\section{RESULTS}

Rats treated with sub-chronic MK-801 were significantly impaired on the standard SOR task, particularly at the longer retention delays (Figure 2a). ANOVA revealed significant effects of group $\left(F_{(1,18)}=5.86, p=0.026\right)$ and delay $\left(F_{(2,36)}=7.06, p=0.003\right)$ on the discrimination ratio measure, but a non-significant interaction $\left(F_{(2,36)}=2.38\right.$, $p=0.107)$. The interpretation of a delay-dependent effect is supported by the results of independent samples $t$-tests on the discrimination ratio scores, which revealed significant novel object preference by both groups at all retention delays (all $p<0.01$ ), except for the MK-801 group at the 24-h delay. Analyses also revealed significant effects of MK-801 treatment on total sample $\left(F_{(1,18)}=7.04, p=0.016\right)$ and choice $\left(F_{(1,18)}=12.71, p=0.002\right)$ exploration measures, as the MK-801 group generally explored slightly less than the control rats (data not shown), but the delay $\times$ group interactions were not significant (sample: $F_{(2,36)}=1.35$, $p=0.27$; choice: $F_{(2,36)}=2.84$, and $\left.p=0.072\right)$.

Rats treated with sub-chronic ketamine were also impaired in standard SOR testing (Figure 2b). ANOVA indicated significant group $\left(F_{(1,18)}=11.38, p=0.003\right)$ and delay $\times$ group interaction $\left(F_{(2,36)}=3.43, p=0.043\right)$ effects on discrimination ratio; the main effect of delay, however, was not significant $\left(F_{(2,36)}=2.57, p=0.09\right)$, and the data in Figure $2 \mathrm{~b}$ illustrate that the control group in this experiment showed no apparent reduction across retention delays. Nonetheless, the delay dependence of the effect in the ketamine-treated rats indicates a mnemonic component to their impairment. Post-hoc analysis of the significant interaction revealed group differences at the $5 \mathrm{~min}(p<0.05)$ and $24 \mathrm{~h}(p<0.001)$ delays only. This pattern was corroborated by the results of independent samples $t$-tests on the discrimination ratio values, which indicated significant novel object preference in all groups and at all delays (all $p<0.05$ ), except for the ketamine-treated rats at the 5-min and 24-h delays. The only significant effect in the analyses of general exploration measures was for the delay $\times$ group interaction in total sample exploration $\left(F_{(2,36)}=5.59, p=0.008\right)$, as the ketamine group explored 

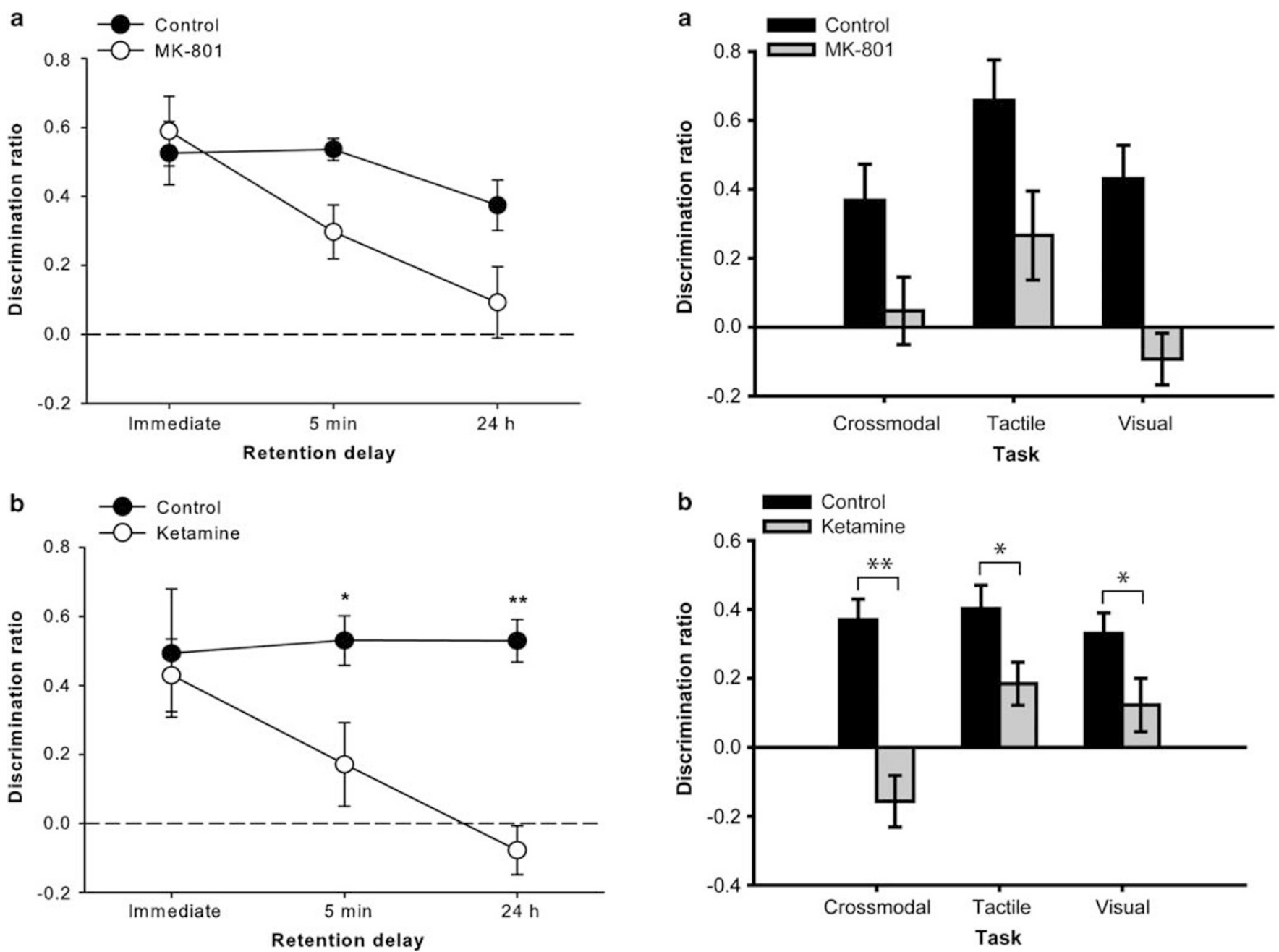

Figure 2 Standard spontaneous object recognition (SOR) task performance (average discrimination ratio $( \pm$ SEM)) by control rats and those treated with sub-chronic (a) MK-80I or (b) ketamine. Rats treated with either drug were significantly impaired compared with saline-treated rats, and this deficit tended to be greater with longer retention delays. ${ }^{*} p<0.05$; *** $p<0.01$.

slightly more than controls in the sample phase for immediate delay trials and slightly less on 5-min trials (data not shown). Post-hocs, however, revealed no significant group differences for sample exploration at any single retention delay.

Sub-chronic MK-801 treatment disrupted performance in the CMOR task and both unimodal (tactile and visual) SOR tasks when a 1-h retention delay was used (Figure $3 \mathrm{a}$ ). ANOVA revealed significant effects of group $\left(F_{(1,17)}=28.16\right.$, $p<0.001)$ and task $\left(F_{(2,34)}=4.43, p=0.02\right)$ on the discrimination ratio measure, but the interaction was not significant $\left(F_{(2,34)}=0.46, p=0.63\right)$. Independent samples $t$-tests on the discrimination ratio scores revealed significant novel object preference in the control group on all tasks (all $p<0.01)$; conversely, the MK-801 animals failed to discriminate between familiar and novel objects on all three tasks with the 1-h delay. Although there were significant differences between tasks in terms of general object exploration (total sample and total choice object exploration), with tactile-only exploration consistently higher than

Figure 3 Cross-modal and unimodal object recognition performance with a I-h retention delay by control rats and those treated with subchronic (a) MK-80I or (b) ketamine. Sub-chronic treatment with either drug caused impairment in all three tasks. $* p<0.05$; ${ }^{*} p<0.01$.

visual-only exploration, the group and task $\times$ group interaction terms were not significant. This was also the case for all subsequent experiments, indicating no gross abnormalities in general object exploratory behavior in the MK-801 and ketamine-treated animals; these data will therefore not be discussed further.

Sub-chronic ketamine treatment similarly impaired CMOR and unimodal SOR task performance with the 1-h retention delay (Figure $3 \mathrm{~b}$ ). ANOVA indicated significant effects of group $\left(F_{(1,38)}=33.28, p<0.001\right)$ and task $\left(F_{(2,76)}=3.95, p=0.023\right)$ on discrimination ratio, as well as a significant task $\times$ group interaction $\left(F_{(2,76)}=3.62\right.$, $p=0.031)$. Post-hoc analysis revealed significant group differences on the tactile $(p<0.05)$, visual $(p<0.05)$, and CMOR $(p<0.001)$ tasks. Moreover, independent samples $t$-tests on the discrimination ratios indicated significant novel object preference in the control group on all tasks (all $p<0.001$ ), as well as for the ketamine group on the tactile SOR task $(p<0.01)$, but not the visual or CMOR tasks.

Given the literature on perturbed multisensory integration function in patients with schizophrenia, and the greater 
absolute CMOR task impairment displayed by the ketamine group with the 1 -h retention delay, we predicted that CMOR task performance might be more sensitive to cognitive dysfunction in these animals than either the standard SOR task or the unimodal versions. This hypothesis was assessed by testing rats in CMOR and unimodal SOR tasks with the immediate retention delay.

Rats treated with sub-chronic ketamine were selectively impaired on the CMOR task when tested with the immediate delay (Figure 4a). Although the group effect was not significant $(F<1)$, there were significant task $\left(F_{(2,36)}=3.52, \quad p=0.04\right)$ and task $\times$ group interaction $\left(F_{(2,36)}=4.17, p=0.024\right)$ effects on the discrimination ratio measure. Post-hocs revealed a significant group difference only on the CMOR task $(p<0.01)$, and independent samples $t$-tests on the discrimination ratio scores indicated that the only condition in which significant novel object preference was not shown was when the ketamine group was tested on the CMOR task. Additional CMOR trials with this batch of rats revealed that systemic nicotine reversed the impairment in the ketamine group (Figure $4 \mathrm{~b}$ ). The group
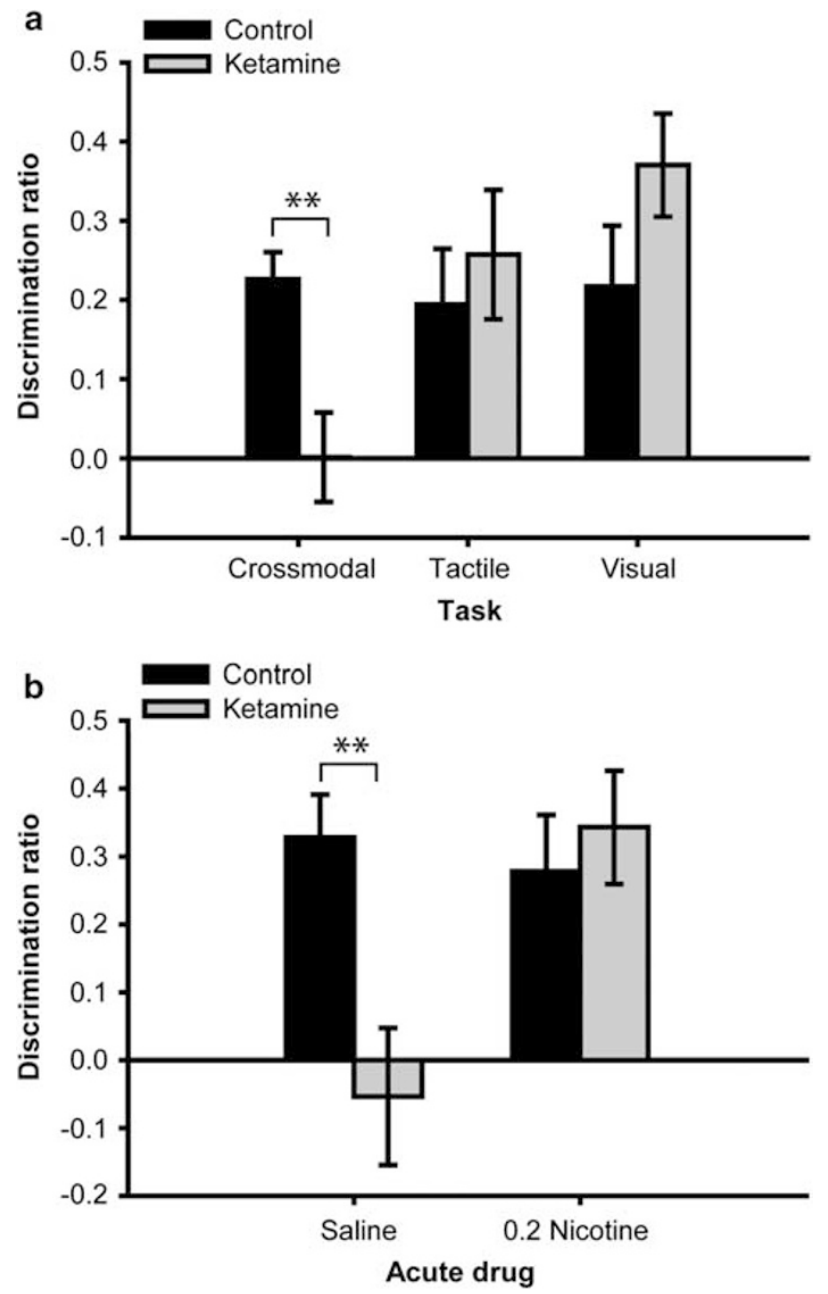

Figure 4 Performance of control and ketamine groups on (a) Crossmodal and unimodal object recognition tasks with an immediate retention delay and (b) additional immediate delay cross-modal trials with acute presample injections of systemic saline or nicotine $(0.2 \mathrm{mg} / \mathrm{kg})$. The selective cross-modal impairment in the ketamine group was reversed by acute nicotine. ${ }^{*} p<0.01$.
$\left(F_{(1,17)}=5.22, \quad p<0.05\right)$ and drug $\times$ group interaction $\left(F_{(1,17)}=5.13, p<0.05\right)$ effects were both significant. Posthoc analysis revealed that the ketamine group performed significantly worse than the control group when given acute saline $(p<0.01)$, but there was no difference when acute nicotine was administered. Moreover, only the ketamine group under acute saline failed to show significant novel object preference. There was also a main effect of drug for total sample exploration $\left(F_{(1,17)}=9.74, p<0.01\right)$, as all rats explored slightly more under acute saline than nicotine. Finally, the drug $\times$ group interaction was significant $\left(F_{(1,17)}=7.49, p<0.05\right)$ for choice exploration, and posthocs indicated that this was due to control animals exploring more on saline than nicotine trials $(p<0.05)$; independent samples $t$-tests, however, indicated no differences between groups within the different acute drug conditions.

Rats treated sub-chronically with MK-801 were similarly significantly impaired relative to controls when tested with the immediate delay (Figure 5a). ANOVA on the discrimination ratio data indicated significant group $\left(F_{(1,17)}=5.29\right.$, $p=0.034)$, task $\left(F_{(2,34)}=7.07, p=0.003\right)$, and interaction terms $\left(F_{(2,34)}=4.01, p=0.027\right)$. Post-hoc analysis of the interaction revealed a significant group difference only on the CMOR task $(p<0.01)$. In further support of this interactive effect, independent samples $t$-tests indicated significant novel object preference for each group on each task (all $p<0.05$ ), except for the MK-801 rats when tested on the CMOR task. When this same batch of rats was tested on additional CMOR trials with the immediate delay, systemic nicotine reversed the impairment in the MK-801 group (Figure 5b). Although the group effect was not significant $(F<1)$, there were significant drug $\left(F_{(1,17)}=19.88, \quad p<0.001\right)$ and drug $\times$ group interaction $\left(F_{(1,17)}=30.56, p<0.001\right)$ effects. Post-hoc analysis revealed that the MK-801 group performed significantly worse than the control group under acute saline treatment $(p<0.05)$, but significantly better when both were administered acute systemic nicotine $(p<0.01)$. Only the MK-801 group under acute saline failed to show significant novel object preference. There were no significant effects in the analyses of general exploration measures from the sample or choice phases.

The ability of nicotine to reverse CMOR impairments in MK-801-treated rats was further explored in a new group of animals. Nicotine dose-dependently rescued CMOR performance of the MK-801 group when tested with the immediate retention delay (Figure $5 \mathrm{c}$ ). ANOVA on the discrimination ratio data indicated that, although both group $\left(F_{(1,17)}=0.19\right.$, $p=0.667)$ and drug $\left(F_{(3,51)}=1.92, p=0.137\right)$ main effects were nonsignificant, a significant interaction effect was found $\left(F_{(3,51)}=4.44, p=0.008\right)$. Post-hoc analysis of the interaction revealed a significant group difference only when rats received an acute injection of saline $(p<0.05)$. Furthermore, independent samples $t$-tests on the discrimination ratios indicated significant novel object preference for the control group when administered 0, 0.05, and $0.2 \mathrm{mg} / \mathrm{kg}$ nicotine $(0.01,0.05$, and $p<0.01$, respectively) and for the MK-801 group when receiving 0.2 and $0.8 \mathrm{mg} / \mathrm{kg}$ nicotine ( 0.05 and $p<0.01$, respectively). Additional paired samples $t$-tests indicated that, within the MK-801 group, CMOR task performance following acute administration of 


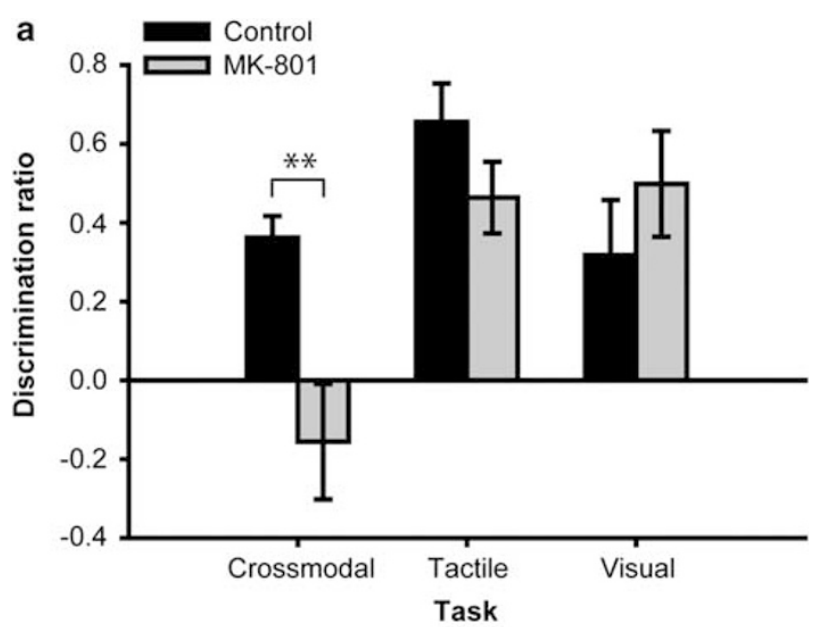

$0.8 \mathrm{mg} / \mathrm{kg}$ nicotine was significantly better than with acute injections of $0.05 \mathrm{mg} / \mathrm{kg}$ nicotine $(p<0.05)$ or saline $(p<0.05)$; the $\mathrm{MK}-801$ group performance with $0.2 \mathrm{mg} / \mathrm{kg}$ nicotine was also significantly better than with saline $(p<0.05)$; all other paired samples $t$-tests within the two groups were nonsignificant. Analyses also revealed significant effects of nicotine treatment on total sample exploration $\left(F_{(3,51)}=4.43, p=0.008\right)$, indicating slightly less object exploration in the 0.2 and $0.8 \mathrm{mg} / \mathrm{kg}$ nicotine conditions compared with acute saline (data not shown). Importantly, however, this effect was similar in both the control and MK801 groups, as the drug $\times$ group interaction was not significant $\left(F_{(3,51)}=0.234, p=0.872\right)$.

As a general note, systemic MK-801 and ketamine are typically associated with acute motoric effects (Gilmour et al, 2009; Smith et al, 2011). Indeed, such effects were often observed in the first $10-15 \mathrm{~min}$ following injections during the sub-chronic administration period. However, these motoric effects did not appear to persist beyond the drug injection phase for MK-801 or ketamine. Qualitative observations and the absence of consistent significant group differences in terms of the general sample and choice phase exploratory measures described above were consistent with the interpretation that the acute motoric effects of MK-801 and ketamine did not extend into the object recognition testing phases.

\section{DISCUSSION}

Few studies have directly assessed multisensory integration in schizophrenia. Recent research, however, reveals abnormal multisensory processing in schizophrenic patients (Stone et al, 2011; Williams et al, 2010). This study represents a valuable extension of current animal models by providing a novel method for evaluating this potentially overlooked aspect of cognitive dysfunction in schizophrenia. Indeed, our results indicate that the CMOR task is highly sensitive to cognitive deficits in rats treated with subchronic NMDAR antagonists. Rats treated sub-chronically with MK-801 or ketamine were impaired on the standard SOR task with intermediate (5-min) and long (24-h) retention delays, but not when the delay was substantially shortened to reduce mnemonic demands. These animals were also impaired when tested with cross-modal and unimodal (tactile or visual) object recognition tasks using a 1-h retention delay. The impairment in the unimodal tasks, however, as in the standard SOR task, disappeared when the retention delay was reduced. Conversely, the CMOR task impairment persisted even at the minimal delay. This selective CMOR deficit was dose-dependently reversed by acute nicotine injections. The overall patterns of results in MK-801- and ketamine-treated rats were highly similar, further strengthening our interpretation of a robust CMOR deficit in rats treated sub-chronically with NMDAR antagonists. These findings may have important ramifications for understanding the specific cognitive impairments affecting patients with schizophrenia, as well as for developing successful treatment strategies.

Sub-chronic treatment with NMDAR antagonists produces robust object recognition memory impairments in rats (Lyon et al, 2011), and the current results replicate and 
extend this established finding. These results speak to the severity of object recognition disruption in such animals by showing that they are significantly impaired in unimodal (tactile or visual) versions of the standard SOR task. The inclusion of these unimodal tasks in such a study is important, as they help to control for aspects of the CMOR task not directly related to multisensory integration. The unimodal deficits, like the standard SOR impairment, may be predominantly mnemonic in nature, as they were no longer apparent when memory demands were minimized. Conversely, the CMOR deficit was observed even when the immediate retention delay was used, suggesting a disruption in multisensory integration - the key difference between the CMOR and unimodal tasks-even with little mnemonic demand. Although the switch from dark to light conditions is a necessary additional procedural difference between the unimodal and cross-modal versions of the task, this does not seem to have had a major role in the deficit observed, as no reliable changes in general exploratory behavior were seen in immediate delay CMOR trials relative to unimodal trials. There also were no other obvious differences between the unimodal and CMOR immediate delay trials, as experimenters were careful to equate the delay times and movement of animals from the exploratory area to the start box of the Y-shaped apparatus in all three versions of the task

Although it is possible that the various unimodal, SOR, and CMOR task impairments describe here are psychologically and neurobiologically independent, it is tempting to view these deficits as different manifestations of the same underlying neurobiological dysfunction. There is ample evidence that sub-chronic NMDAR antagonist treatment affects parvalbumin-containing GABAergic inhibitory interneurons in the cortex, and these interneurons may be critical to oscillatory activity central to cognitive functions (Cochran et al, 2003; Nakazawa et al, 2012; Pratt et al, 2008); similar deficiencies are observed in the brains of patients with schizophrenia (Lewis et al, 2012). In the absence of this oscillatory control, synchronous information processing in the cortex may be disrupted. Such cortical disinhibition could be expected to affect brain areas responsible for visual and tactile object memory, as well as their interaction. Our previous results indicate that at least two cortical regions - the perirhinal and posterior parietal corticesmust interact in the service of CMOR task performance (Winters and Reid, 2010), and recent data from our lab indicate that the orbitofrontal region of the prefrontal cortex may also contribute to this task (JM Reid, DL Jacklin, and BD Winters, unpublished observations). Thus, although cortical disinhibition could disrupt basic object memory processing, it might be expected to have a larger impact on performance in the CMOR task, which likely relies on even greater integration of information processing across cortical regions. The neural effects induced by sub-chronic NMDAR antagonists are widespread (Abdul-Monim et al, 2007; Keilhoff et al, 2004; Mouri et al, 2007), and the extent to which dysfunction in various cortical and/or subcortical regions contributes to the selective CMOR deficits observed here remains to be determined. Ongoing and future studies in our lab will aim to answer these and related questions.

The cholinergic system is a potentially important target in the development of novel pharmacological treatments for schizophrenia symptoms. Indeed, studies have shown that drugs activating muscarinic or nicotinic acetylcholine receptor (nAChR) subtypes have potential for the treatment of cognitive symptoms in patients with schizophrenia (for review, see Jones et al, 2012). Specifically, nicotine, as well as agonists and positive allosteric modulators of $\alpha 7$ and $\alpha 4 \beta 2 \mathrm{nAChRs}$, attenuate various cognitive deficits in rodent models of schizophrenia (Boess et al, 2007; Hashimoto et al, 2008; Hauser et al, 2009; Hurst et al, 2005; Pichat et al, 2007; Rushforth et al, 2011; Thomsen et al, 2009; Timmermann et al, 2007; Wallace et al, 2011; Wildeboer and Stevens, 2008; Wishka et al, 2006). Our observations that nicotine reverses the selective CMOR impairment in rats treated with subchronic NMDAR antagonists is consistent with studies using standard object recognition tasks and extends these findings by implicating $\mathrm{nAChRs}$ in the potential treatment of multisensory integration deficits in schizophrenia.

The dose-response experiment confirmed that the facilitation of CMOR performance by nicotine was dosedependent, at least in MK-801-treated rats, which were significantly worse than controls when treated with saline but not when administered acute nicotine. The enhancing effect of nicotine was strongest at the intermediate $(0.2 \mathrm{mg} / \mathrm{kg})$ and highest $(0.8 \mathrm{mg} / \mathrm{kg})$ doses, the only conditions in which MK-801 rats demonstrated a significant discrimination between novel and familiar objects. The dose-response experiment also enabled us to assess the effects of nicotine in naïve rats, as the earlier nicotine trials were run with animals that had been repeatedly tested on other forms of the object recognition tasks. Such retesting could conceivably impact the effects of acute drugs in subsequent trials. For instance, it is possible that the apparent hypersensitivity of MK-801 rats to acute nicotine in the single-dose experiment could be related to the extensive testing experienced by these rats before nicotine trials. Thus, we prepared a new batch of MK-801 rats to assess the effects of acute nicotine in animals not previously tested. The doseresponse data, however, while not identical to the results seen in the single-dose nicotine experiment, indicate that naïve MK-801 rats may nonetheless display a heightened or shifted behavioral response to acute nicotine. The $0.2 \mathrm{mg} / \mathrm{kg}$ dose of nicotine improved MK-801 CMOR task performance back to control levels, but, unlike in the previous experiment, MK-801 rats were not significantly better than controls. However, a result similar to this earlier finding was seen at the $0.8 \mathrm{mg} / \mathrm{kg}$ dose of nicotine, as the performance of MK-801 rats remained significantly above chance, whereas controls treated with the same dose of nicotine did not discriminate between novel and familiar objects. We are not aware of any similar findings in the literature, and future studies will be required to ascertain the basis and robustness of this apparent hypersensitivity to nicotine in MK-801-treated animals performing the CMOR task. Additional dose-response analyses should also be run to test the possibility that ketamine-treated rats display a similar pattern of altered responsivity to nicotine. Future work is also required to determine specific nAChR subtype contributions to the current effects. Furthermore, there is ample evidence for interactions between nicotinic activation and other neurotransmitters, such as glutamate and GABA (Levin et al, 2006; Timofeeva and Levin, 2011); thus, another goal of future research will be to assess the potential 
involvement of glutamatergic or GABAergic modulation in the facilitative effects of nicotine on CMOR task performance in rats treated sub-chronically with NMDAR antagonists.

Rats are capable of recognizing objects across sensory modalities in the CMOR task, and our results indicate that this task is acutely sensitive to the cognitive impairment present in rats treated sub-chronically with non-competitive NMDAR antagonists. Moreover, the CMOR deficit was apparent with testing parameters under which other object recognition impairments disappeared, suggesting a relatively selective and severe disruption of multisensory integration in these animals. Given recent findings highlighting altered multisensory processing in patients with schizophrenia (Stone et al, 2011; Williams et al, 2010), there is a current need for more refined methods to evaluate this aspect of cognition in animal models. The CMOR task represents a flexible and easily implemented addition to established testing batteries that appears to tap a cognitive domain not accessed by typical object recognition tasks. As no single preparation comprehensively recapitulates the full spectrum of schizophrenic symptoms, testing other animal models, including genetic (Robertson and Feng, 2011) and developmental (Brown, 2011) models, in the CMOR task should provide greater insight into the biological bases of this specific cognitive impairment and how it relates to schizophrenia. The current results, however, are likely to be highly relevant to schizophrenia considering the established relationships between NMDAR-mediated intracellular pathways and schizophrenia-related genetic factors (Harrison and Weinberger, 2005). The current results therefore not only implicate NMDAR hypofunction in multisensory integration deficits, but also demonstrate the valuable potential of the CMOR task to facilitate future systematic study of the etiology and treatment of cognitive impairment in schizophrenia.

\section{ACKNOWLEDGEMENTS}

This research was supported by a National Sciences and Engineering Research Council (NSERC) of Canada Discovery Grant to BDW and an Ontario Graduate Scholarship to DLJ.

\section{DISCLOSURE}

The authors declare no conflict of interest.

\section{REFERENCES}

Abdul-Monim Z, Neill JC, Reynolds GP (2007). Sub-chronic psychotomimetic phencyclidine induces deficits in reversal learning and alterations in parvalbumin-immunoreactive expression in the rat. J Psychopharmacol 21: 198-205.

Adler LE, Pachtman E, Franks RD, Pecevich M, Waldo MC, Freedman R (1982). Neurophysiological evidence for a defect in neuronal mechanisms involved in sensory gating in schizophrenia. Biol Psychiatry 17: 639-654.

Beninger RJ, Forsyth JK, Van Adel M, Reynolds JN, Boegman RJ, Jhamandas K (2009). Subchronic MK-801 behavioural deficits in rats: partial reversal by the novel nitrate GT 1061. Pharmacol Biochem Behav 91: 495-502.
Boess FG, De Vry J, Erb C, Flessner T, Hendrix M, Luithle J et al (2007). The novel alpha7 nicotinic acetylcholine receptor agonist $\mathrm{N}$-[(3R)-1-azabicyclo[2.2.2]oct-3-yl]-7-[2-(methoxy)phenyl]-1benzofuran-2- carboxamide improves working and recognition memory in rodents. J Pharmacol Exp Ther 321: 716-725.

Braff DL (1989). Sensory input deficits and negative symptoms in schizophrenic patients. Am J Psychiatry 146: 1006-1011.

Brown AS (2011). Exposure to prenatal infection and risk of schizophrenia. Front Psychiatry 2: 63.

Butler PD, Silverstein SM, Dakin SC (2008). Visual perception and its impairment in schizophrenia. Biol Psychiatry 64: 40-47.

Cochran SM, Kennedy M, McKerchar CE, Steward LJ, Pratt JA, Morris BJ (2003). Induction of metabolic hypofunction and neurochemical deficits after chronic intermittent exposure to phencyclidine: differential modulation by antipsychotic drugs. Neuropsychopharmacology 28: 265-275.

de Gelder B, Vroomen J, Annen L, Masthof E, Hodiamont P (2003). Audio-visual integration in schizophrenia. Schizophr Res 59: 211-218.

de Gelder B, Vroomen J, de Jong SJ, Masthoff ED, Trompenaars FJ, Hodiamont P (2005). Multisensory integration of emotional faces and voices in schizophrenics. Schizophr Res 72: 195-203.

de Jong JJ, Hodiamont PP, Van den Stock J, de Gelder B (2009). Audiovisual emotion recognition in schizophrenia: reduced integration of facial and vocal affect. Schizophr Res 107: 286-293.

Enomoto T, Floresco SB (2009). Disruptions in spatial working memory, but not short-term memory, induced by repeated ketamine exposure. Prog Neuropsychopharmacol Biol Psychiatry 33: 668-675.

Enomoto T, Noda Y, Mouri A, Shin EJ, Wang D, Murai R et al (2005). Long-lasting impairment of associative learning is correlated with a dysfunction of $\mathrm{N}$-methyl-D-aspartate-extracellular signaling-regulated kinase signaling in mice after withdrawal from repeated administration of phencyclidine. Mol Pharmacol 68: 1765-1774.

Ford JM, Gray M, Whitfield SL, Turken AU, Glover G, Faustman WO et al (2004). Acquiring and inhibiting prepotent responses in schizophrenia: event-related brain potentials and functional magnetic resonance imaging. Arch Gen Psychiatry 61: 119-129.

Forwood SE, Winters BD, Bussey TJ (2005). Hippocampal lesions that abolish spatial maze performance spare object recognition memory at delays of up to $48 \mathrm{~h}$. Hippocampus 15: 347-355.

Gilmour G, Dix S, Fellini L, Gastambide F, Plath N, Steckler T et al (2011). NMDA receptors, cognition and schizophrenia-Testing the validity of the NMDA receptor hypofunction hypothesis. Neuropharmacology 62: 1401-1412.

Gilmour G, Pioli EY, Dix SL, Smith JW, Conway MW, Jones WT et al (2009). Diverse and often opposite behavioural effects of NMDA receptor antagonists in rats: implications for "NMDA antagonist modeling" of schizophrenia. Psychopharmacology 205: 203-216.

Harrison PJ, Weinberger DR (2005). Schizophrenia genes, gene expression, and neuropathology: on the matter of their convergence. Mol Psychiatry 10: 40-68.

Hashimoto K, Ishima T, Fujita Y, Matsuo M, Kobashi T, Takahagi $\mathrm{M}$ et al (2008). Phencyclidine-induced cognitive deficits in mice are improved by subsequent subchronic administration of the novel selective alpha7 nicotinic receptor agonist SSR180711. Biol Psychiatry 63: 92-97.

Hauser TA, Kucinski A, Jordan KG, Gatto GJ, Wersinger SR, Hesse RA et al (2009). TC-5619: an alpha7 neuronal nicotinic receptorselective agonist that demonstrates efficacy in animal models of the positive and negative symptoms and cognitive dysfunction of schizophrenia. Biochem Pharmacol 78: 803-812.

Hurst RS, Hajos M, Raggenbass M, Wall TM, Higdon NR, Lawson JA et al (2005). A novel positive allosteric modulator of the alpha7 neuronal nicotinic acetylcholine receptor: in vitro and in vivo characterization. J Neurosci 25: 4396-4405. 
Itil T, Keskiner A, Kiremitci N, Holden JM (1967). Effect of phencyclidine in chronic schizophrenics. Can Psychiatr Assoc J 12: $209-212$

Jacobs GH, Fenwick JA, Williams GA (2001). Cone-based vision of rats for ultraviolet and visible lights. J Exp Biol 204(Part 14): 2439-2446.

Jentsch JD, Roth RH (1999). The neuropsychopharmacology of phencyclidine: from NMDA receptor hypofunction to the dopamine hypothesis of schizophrenia. Neuropsychopharmacology 20: 201-225.

Jones CK, Byun N, Bubser M (2012). Muscarinic and nicotinic acetylcholine receptor agonists and allosteric modulators for the treatment of schizophrenia. Neuropsychopharmacology 37: $16-42$.

Keilhoff G, Becker A, Grecksch G, Wolf G, Bernstein HG (2004). Repeated application of ketamine to rats induces changes in the hippocampal expression of parvalbumin, neuronal nitric oxide synthase and cFOS similar to those found in human schizophrenia. Neuroscience 126: 591-598.

Leonard S, Breese C, Adams C, Benhammou K, Gault J, Stevens K et al (2000). Smoking and schizophrenia: abnormal nicotinic receptor expression. Eur J Pharmacol 393: 237-242.

Levin ED, McClernon FJ, Rezvani AH (2006). Nicotinic effects on cognitive function: behavioral characterization, pharmacological specification, and anatomic localization. Psychopharmacology (Berl) 184: 523-539.

Lewis DA, Curley AA, Glausier JR, Volk DW (2012). Cortical parvalbumin interneurons and cognitive dysfunction in schizophrenia. Trends Neurosci 35: 57-67.

Lewis DA, Gonzalez-Burgos G (2008). Neuroplasticity of neocortical circuits in schizophrenia. Neuropsychopharmacology 33: 141-165.

Luby ED, Cohen BD, Rosenbaum G, Gottlieb JS, Kelley R (1959). Study of a new schizophrenomimetic drug; sernyl. AMA Arch Neurol Psychiatry 81: 363-369.

Lyon L, Saksida LM, Bussey TJ (2011). Spontaneous object recognition and its relevance to schizophrenia: a review of findings from pharmacological, genetic, lesion and developmental rodent models. Psychopharmacology (Berl) 220: 647-672.

McGurk H, MacDonald J (1976). Hearing lips and seeing voices. Nature 264: 746-748.

Mouri A, Noda Y, Noda A, Nakamura T, Tokura T, Yura Y et al (2007). Involvement of a dysfunctional dopamine-D1/N-methyl$\mathrm{d}$-aspartate-NR1 and $\mathrm{Ca} 2+/$ calmodulin-dependent protein $\mathrm{ki}$ nase II pathway in the impairment of latent learning in a model of schizophrenia induced by phencyclidine. Mol Pharmacol 71: 1598-1609.

Nakazawa K, Zsiros V, Jiang Z, Nakao K, Kolata S, Zhang S et al (2012). GABAergic interneuron origin of schizophrenia pathophysiology. Neuropharmacology 62: 1574-1583.

Neill JC, Barnes S, Cook S, Grayson B, Idris NF, McLean SL et al (2010). Animal models of cognitive dysfunction and negative symptoms of schizophrenia: focus on NMDA receptor antagonism. Pharmacol Ther 128: 419-432.

Nuechterlein KH, Barch DM, Gold JM, Goldberg TE, Green MF, Heaton RK (2004). Identification of separable cognitive factors in schizophrenia. Schizophr Res 72: 29-39.

Pearl D, Yodashkin-Porat D, Katz N, Valevski A, Aizenberg D, Sigler $\mathrm{M}$ et al (2009). Differences in audiovisual integration, as measured by McGurk phenomenon, among adult and adolescent patients with schizophrenia and age-matched healthy control groups. Compr Psychiatry 50: 186-192.

Pichat P, Bergis OE, Terranova JP, Urani A, Duarte C, Santucci V et al (2007). SSR180711, a novel selective alpha7 nicotinic receptor partial agonist: (II) efficacy in experimental models predictive of activity against cognitive symptoms of schizophrenia. Neuropsychopharmacology 32: 17-34.
Pratt JA, Winchester C, Egerton A, Cochran SM, Morris BJ (2008). Modelling prefrontal cortex deficits in schizophrenia: implications for treatment. Br J Pharmacol 153(Suppl 1): S465-S470.

Reynolds GP, Abdul-Monim Z, Neill JC, Zhang ZJ (2004). Calcium binding protein markers of GABA deficits in schizophrenia-postmortem studies and animal models. Neurotox Res 6: $57-61$.

Robertson HR, Feng G (2011). Annual Research Review: Transgenic mouse models of childhood-onset psychiatric disorders. J Child Psychol Psychiatry 52: 442-475.

Ross LA, Saint-Amour D, Leavitt VM, Molholm S, Javitt DC, Foxe JJ (2007). Impaired multisensory processing in schizophrenia: deficits in the visual enhancement of speech comprehension under noisy environmental conditions. Schizophr Res 97: 173-183.

Rushforth SL, Steckler T, Shoaib M (2011). Nicotine improves working memory span capacity in rats following sub-chronic ketamine exposure. Neuropsychopharmacology 36: 2774-2781.

Smith JW, Gastambide F, Gilmour G, Dix S, Foss J, Lloyd K et al (2011). A comparison of the effects of ketamine and phencyclidine with other antagonists of the NMDA receptor in rodent assays of attention and working memory. Psychopharmacology (Berl) 217: 255-269.

Stein BE, Meredith MA (1993). The merging of the senses. MIT Press: Cambridge MA.

Stone DB, Urrea LJ, Aine CJ, Bustillo JR, Clark VP, Stephen JM (2011). Unisensory processing and multisensory integration in schizophrenia: a high-density electrical mapping study. Neuropsychologia 49: 3178-3187.

Thomsen MS, Christensen DZ, Hansen HH, Redrobe JP, Mikkelsen JD (2009). alpha Nicotinic acetylcholine receptor activation prevents behavioral and molecular changes induced by repeated phencyclidine treatment. Neuropharmacology 56: 1001-1009.

Timmermann DB, Gronlien JH, Kohlhaas KL, Nielsen EO, Dam E, Jorgensen TD et al (2007). An allosteric modulator of the alpha7 nicotinic acetylcholine receptor possessing cognition-enhancing properties in vivo. J Pharmacol Exp Ther 323: 294-307.

Timofeeva OA, Levin ED (2011). Glutamate and nicotinic receptor interactions in working memory: importance for the cognitive impairment of schizophrenia. Neuroscience 195: 21-36.

Wallace TL, Callahan PM, Tehim A, Bertrand D, Tombaugh G, Wang S et al (2011). RG3487, a novel nicotinic alpha7 receptor partial agonist, improves cognition and sensorimotor gating in rodents. J Pharmacol Exp Ther 336: 242-253.

Wildeboer KM, Stevens KE (2008). Stimulation of the alpha4beta2 nicotinic receptor by 5-I A-85380 improves auditory gating in DBA/2 mice. Brain Res 1224: 29-36.

Williams LE, Light GA, Braff DL, Ramachandran VS (2010). Reduced multisensory integration in patients with schizophrenia on a target detection task. Neuropsychologia 48: 3128-3136.

Winters BD, Forwood SE, Cowell RA, Saksida LM, Bussey TJ (2004). Double dissociation between the effects of peripostrhinal cortex and hippocampal lesions on tests of object recognition and spatial memory: heterogeneity of function within the temporal lobe. J Neurosci 24: 5901-5908.

Winters BD, Reid JM (2010). A distributed cortical representation underlies crossmodal object recognition in rats. J Neurosci 30: 6253-6261.

Wishka DG, Walker DP, Yates KM, Reitz SC, Jia S, Myers JK et al (2006). Discovery of N-[(3R)-1-azabicyclo[2.2.2]oct-3-yl]furo[2,3-c]pyridine-5-carboxamide, an agonist of the alpha7 nicotinic acetylcholine receptor, for the potential treatment of cognitive deficits in schizophrenia: synthesis and structure- activity relationship. J Med Chem 49: 4425-4436.

Woodruff-Pak DS, Gould TJ (2002). Neuronal nicotinic acetylcholine receptors: involvement in Alzheimer's disease and schizophrenia. Behav Cogn Neurosci Rev 1: 5-20. 